\title{
On the Growth Mechanism of Nickel and Cobalt Nanowires and Comparison of Their Magnetic Properties
}

\author{
T. N. Narayanan ${ }^{1}$, M. M. Shaijumon ${ }^{2}$, Lijie Ci ${ }^{2}$, P. M. Ajayan² $(\varangle)$, and M. R. Anantharaman ${ }^{1}(\bowtie)$ \\ ${ }^{1}$ Department of Physics, Cochin University of Science \& Technology, Cochin-22, Kerala, India \\ ${ }^{2}$ Department of Mechanical Engineering \& Materials Science, Rice University, Houston, TX 77005, USA \\ Received: 22 August 2008 / Revised: 11 October 2008 / Accepted: 28 October 2008 \\ CTsinghua Press and Springer-Verlag 2008. This article is published with open access at Springerlink.com
}

\begin{abstract}
Magnetic nanowires (NWs) are ideal materials for the fabrication of various multifunctional nanostructures which can be manipulated by an external magnetic field. Highly crystalline and textured nanowires of nickel (Ni NWs) and cobalt (Co NWs) with high aspect ratio ( 330) and high coercivity have been synthesized by electrodeposition using nickel sulphate hexahydrate $\left(\mathrm{NiSO}_{4} \cdot 6 \mathrm{H}_{2} \mathrm{O}\right)$ and cobalt sulphate heptahydrate $\left(\mathrm{CoSO}_{4} \cdot 7 \mathrm{H}_{2} \mathrm{O}\right)$ respectively on nanoporous alumina membranes. They exhibit a preferential growth along $\langle 110\rangle$. A general mobility assisted growth mechanism for the formation of $\mathrm{Ni}$ and Co NWs is proposed. The role of the hydration layer on the resulting one-dimensional geometry in the case of potentiostatic electrodeposition is verified. A very high interwire interaction resulting from magnetostatic dipolar interactions between the nanowires is observed. An unusual low-temperature magnetisation switching for field parallel to the wire axis is evident from the peculiar high field $M(T)$ curve.
\end{abstract}

\section{KEYWORDS}

Magnetic nanowires, mobility assisted growth, magnetostatic interaction, electrodeposition, magnetisation switching

\section{Introduction}

Magnetism is a cooperative phenomenon and is dictated by size, dimension, shape, structure, and morphology of the constituent phases along with the type and strength of the magnetic coupling that exists between the constituent phases [1-3]. Nanoscale magnetic materials have been receiving much attention due to their unique magnetic properties that are different from their bulk counterparts, and are promising candidates for various applications. Ferromagnetic nanowires of $\mathrm{Fe}, \mathrm{Co}$, and $\mathrm{Ni}$ are candidate materials for studying fundamental phenomena like micromagnetic reversal processes and quantum size effects [4-6]. Ferromagnetic nanowires and nanotubes have innumerable applications in areas such as ultrahigh density recording [7, 8], GMR sensors [9], supermolecular architectures [10], and nanoscale electronic and optoelectronic devices [11, 12]. Moreover, control of morphology in nanostructures is very important in tailoring their properties [13].

Template assisted synthesis is developing into an elegant chemical approach for the fabrication of nanoscale structures, as an alternative to sophisticated lithographical methods [14], especially

Address correspondence to M. R. Anantharaman,mraiyer@yahoo.com; P. M. Ajayan, ajayan@rice.edu

\section{黑 Springer}


for larger areas. Here, electrodeposition is receiving much attention in the fabrication of magnetic nanostructures because of its low cost, simplicity of operation, and the ability to tailor magnetic properties by tuning the length and diameter of the porous material. These nanowires are promising candidates for nanoscopic electrodes in applied electrochemistry $[15,16]$ and for various other fundamental studies [17-19].

The very high ordering and the magnetic nature of the wires will induce outstanding cooperative phenomena that differ from the bulk and even from their thin film counterparts. Among these ordered magnetic wires, the interwire interactions play an important role and have been the subject of extensive investigations [20-23]. It has been reported that these interwire interactions are so strong that they can even change the easy axis of magnetisation and control the magnetisation reversal, depending upon the strength of the interaction [21-23]. These studies indicate that the modes of magnetisation reversal and other magnetic properties in such systems are strongly influenced by the microstructure and the interwire separation between nanowires. A complete understanding of the mechanism of magnetisation reversal in such systems remains elusive and it is persisting as a challenge for researchers.

In our earlier work, we fabricated cobalt nanotubes (Co NTs) and thick walled Co NTs using template assisted electrodeposition employing acetate precursors. An understanding of the growth mechanism will help in the design of various heterogeneous structures and hybrid multifunctional nanostructures [24], and a mechanism for the formation of Co NTs using acetate precursors was proposed based on mobility assisted growth [25]. In the case of Co NTs a 5-h electrodeposition of cobalt acetate resulted in the formation of 50 $\mu \mathrm{m}$ long nanotubes. Co NTs were obtained when anhydrous cobalt acetate was used as precursor for electrodeposition while thick walled nanotubes were obtained when the cobalt acetate precursor was replaced by cobalt acetate tetrahydrate. Thus the role of the hydration layer in acetate precursors on the resultant one-dimensional structure was established. In this paper, an extension of these studies to the fabrication of nickel and cobalt nanowires using sulphate precursors, instead of the acetate precursors which were previously used for the preparation of Co NTs, is reported. Moreover, the veracity of the mechanism based on mobility assisted growth is tested for the preparation of NWs using sulphate precursors. A comparative study of these two systems, namely Co and Ni NWs, should shed more light on the magnetic properties of these two nanostructured systems.

\section{Experimental}

Alumina membranes (Whatman, $99.9 \%$ pure) having an average pore diameter of $150 \mathrm{~nm}$ and uniform pore density were used for electrodeposition. Electrochemical deposition was carried out in the nanopores, using a standard three-electrode potentiostat system (Princeton EG\&G 273A). One side of the alumina membrane was coated by vacuum evaporation with silver $(\mathrm{Ag}, \sim 200 \mathrm{~nm}$ thickness), which acted as the working electrode for electrodeposition. The reference electrode was $\mathrm{Ag} / \mathrm{AgCl}$ and platinum was used as the counter electrode. For Ni NWs, an aqueous solution of 0.2 $\mathrm{mol} / \mathrm{L}$ nickel sulphate hexahydrate $\left(\mathrm{NiSO}_{4} \cdot 6 \mathrm{H}_{2} \mathrm{O}\right)$ in $0.1 \mathrm{~mol} / \mathrm{L}$ boric acid $\left(\mathrm{H}_{3} \mathrm{BO}_{3}\right)$ was used as the precursor; the latter works as a buffer. Co NWs were fabricated using an aqueous solution of $0.2 \mathrm{~mol} / \mathrm{L}$ $\mathrm{CoSO}_{4} \cdot 7 \mathrm{H}_{2} \mathrm{O}$ in $0.1 \mathrm{~mol} / \mathrm{L}$ boric acid $\left(\mathrm{H}_{3} \mathrm{BO}_{3}\right)$. The $\mathrm{X}$-ray diffraction $(\mathrm{XRD})$ patterns of nanowires embedded in the alumina templates were recorded using $\mathrm{Cu} \mathrm{K} \alpha$ radiation, $\lambda=1.5418 \AA$ (Rigaku Dmax-C). Field emission scanning electron microscopy (JSM-6335 FESEM) was employed to study the morphology of nanowires.

After electrodeposition of nickel $(\mathrm{Ni})$ or cobalt (Co), the working electrode (silver, Ag) was etched by one or two drops of concentrated nitric acid. Individual nanowires were separated by etching out the alumina using $3 \mathrm{~mol} / \mathrm{L}$ sodium hydroxide $(\mathrm{NaOH})$ solution and decanting the dissolved alumina using magnetic separation. These nanowires were subjected to further studies. Transmission electron microscopy (TEM) experiments were performed using a JEM 2010 transmission electron microscope. Magnetisation measurements were 
carried out using a SQUID magnetometer (MPMS5S XL Quantum Design) by keeping the nanowires inside the alumina pores in order to retain the alignment intact.

\section{Results and discussion}

\subsection{Growth mechanism of nanowires}

Figure 1(a) shows a typical time dependence of electrical current for Ni NWs during deposition. The horizontal part of the current transient (Fig. 1(a)) indicates the production of nanowires in the pores of the alumina membrane. Current variation also shows that there is no over-deposition and that the nanowire is in the growth state [26]. Figure 1(b) is a schematic of the formation mechanism of the nanowires. This is based on a model involving mobility assisted growth [25].

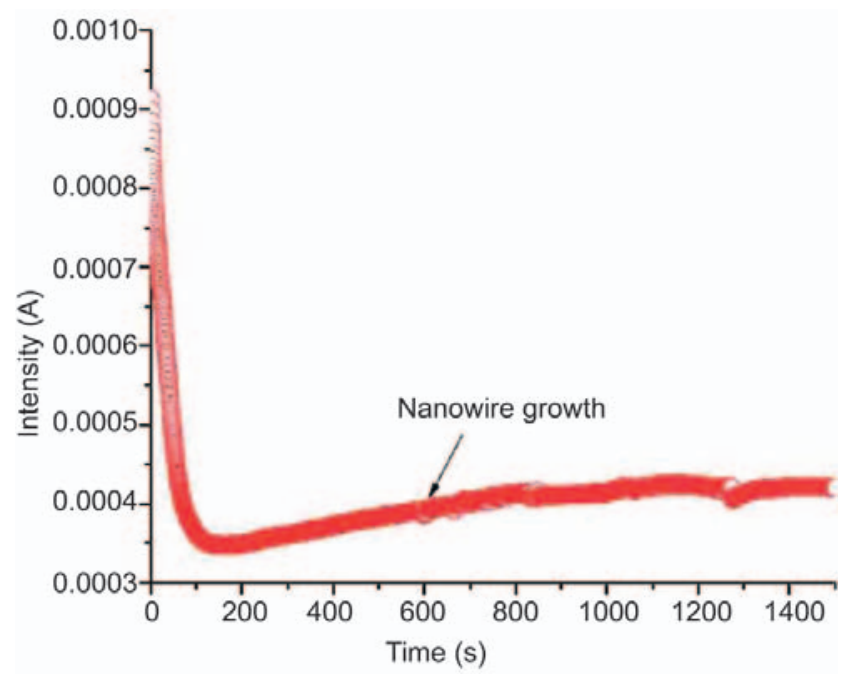

(a)

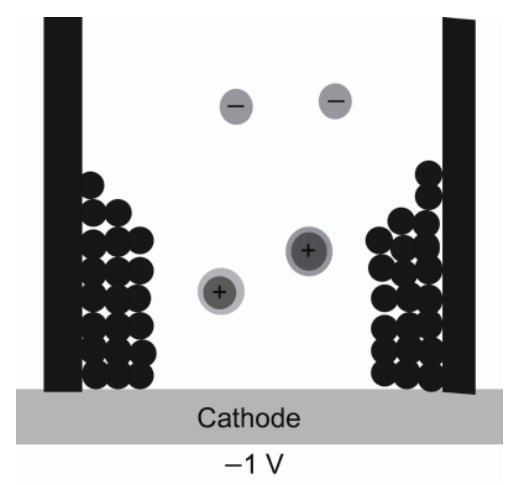

(b)

Figure 1 (a) Current-time curve for electrodeposition of $\mathrm{Ni}$; (b) schematic illustration of nanowire growth
It is a well established fact that growth during electrodeposition in porous materials originates from the cathode surface at the bottom edge of the pore. The high surface area and presence of sites with low coordination number in the porous part of the alumina afford energetically favourable sites for initiating metal adsorption during electrodeposition. Electrodeposition voltage was optimized for both precursors $\left(\mathrm{NiSO}_{4} \cdot 6 \mathrm{H}_{2} \mathrm{O}\right.$ and $\left.\mathrm{CoSO}_{4} \cdot 7 \mathrm{H}_{2} \mathrm{O}\right)$ as $-1 \mathrm{~V}$ (potentiostatic). When this negative potential $(-1 \mathrm{~V})$ is applied to the working electrode, divalent metal ions of $\mathrm{Ni}^{2+}$ or $\mathrm{Co}^{2+}$ surrounded by their hydration layers move towards the cathode and are reduced to the metal. The movement rate of ions in a given electric field $E$ depends on two factors, namely, the mobility of the ions and the potential gradient across the working and counter electrodes,

$$
\begin{aligned}
& V_{+}=\mu_{+} \mathrm{d} E / \mathrm{d} x \\
& V_{-}=\mu_{-} \mathrm{d} E / \mathrm{d} x
\end{aligned}
$$

where $V_{+}, V_{-}$are the movement rates of cobalt cations and sulphate anions and $\mu_{+}, \mu_{-}$are the mobilities of cobalt and sulphate ions, respectively. The components $V_{\|}$and $V_{\perp}$ of the metal ion movement rate $\left(V_{+}\right)$, and the competition between them are the key parameters determining the resultant geometry after electrodeposition.

During the electrodeposition process, $\mathrm{Ni}^{2+}$ ions in the $\mathrm{NiSO}_{4} \cdot 6 \mathrm{H}_{2} \mathrm{O}$ (or $\mathrm{Co}^{2+}$ ions in the case of $\mathrm{CoSO}_{4} \cdot 7 \mathrm{H}_{2} \mathrm{O}$ ) precursor are surrounded by a hydration shell. This hydration layer will effectively reduce the metal ion mobility, and the components of metal ion velocity become almost equal $\left(V_{\|} \approx V_{\perp}\right)$. The rate of reduction of metal ions thus becomes equal in both directions, resulting in nanowires. This growth mechanism is identical to the one we observed in Co NTs where acetate precursors were used for electrodeposition [25].

The XRD pattern of Ni NWs (Fig. 2(a)) indicates that the wires are highly crystalline (polycrystalline) in nature and that they crystallize in the face centered cubic (fcc) phase. Preferential growth along (220) can also be noticed from the XRD pattern. Figure 2(b) shows the XRD pattern of Co NWs. The formation of a highly crystalline (polycrystalline) and textured Co hexagonal close packed (hcp) phase is evident from the XRD. It must be noted here that the broad diffraction peak occurring around $15^{\circ}-35^{\circ}$ is due to

\section{圈国 Springer}




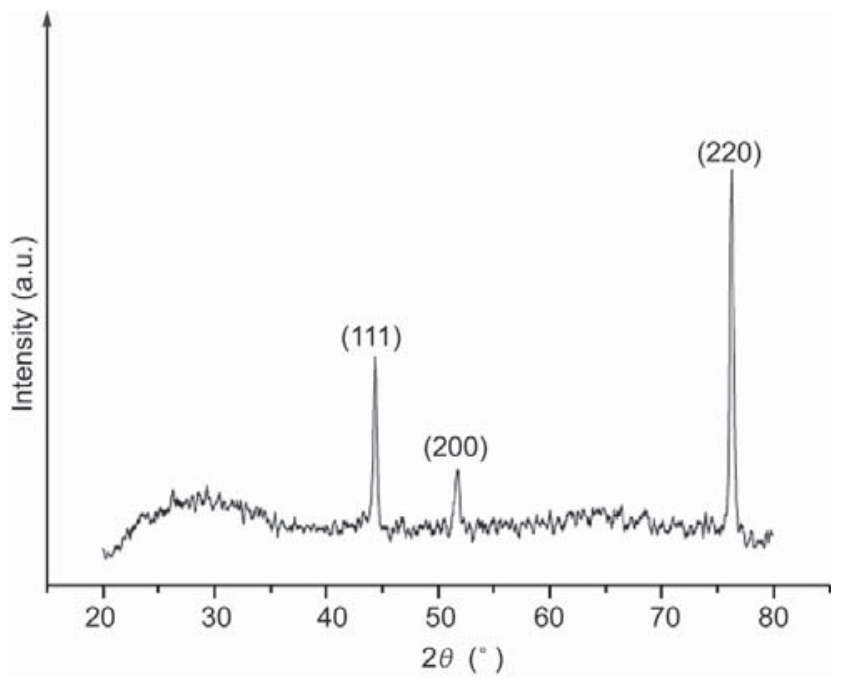

(a)

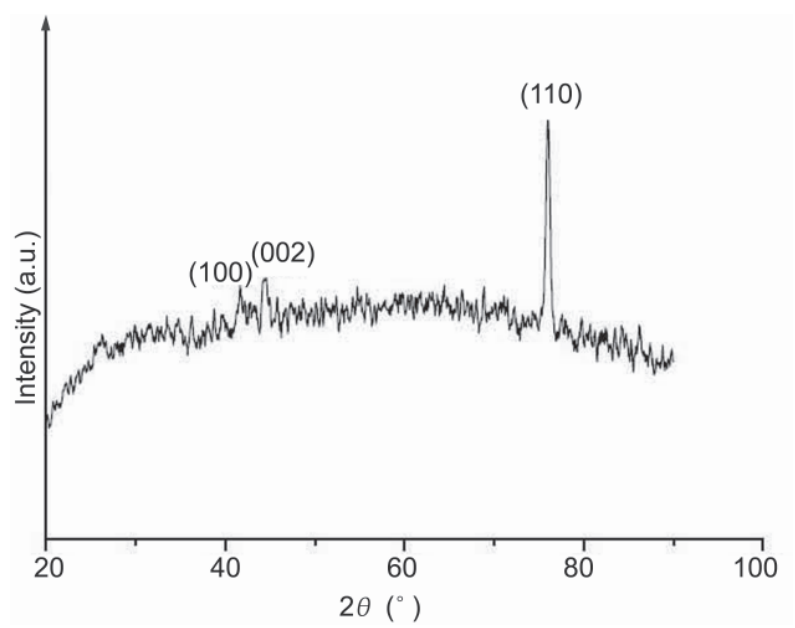

(b)

Figure 2 (a) XRD pattern of electrodeposited nickel; (b) XRD pattern of electrodeposited cobalt

amorphous alumina [27].

The high relative intensities of the 220 and 111 diffraction lines in the case of Ni NWs is evidence for strong texturing along the $\langle 110\rangle$ direction [28]. FESEM images (Figs. 3(a), 3(b), and 3(c)) indicate the formation of good quality nanowires with a maximum length of $50 \mu \mathrm{m}$ and diameter of $150 \mathrm{~nm}$. Figures 3(a) and 3(c) depict the bundles of nanowires of $\mathrm{Ni}$ and Co respectively obtained after the removal of alumina by alkaline treatment. Figure $3(\mathrm{~b})$ shows individual Ni NWs on a silicon substrate.

Figures 4(a) and 4(b) illustrate the composition of nanowires using energy dispersive spectroscopy (EDS). The purities of $\mathrm{Ni}$ and Co NWs are confirmed by EDS and it is to be noted that there

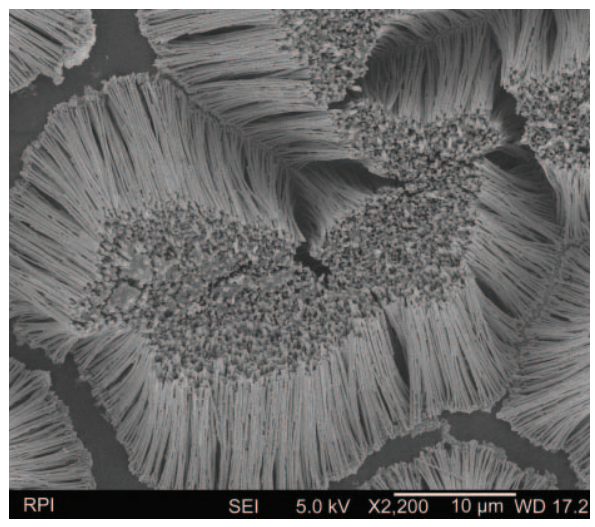

(a)

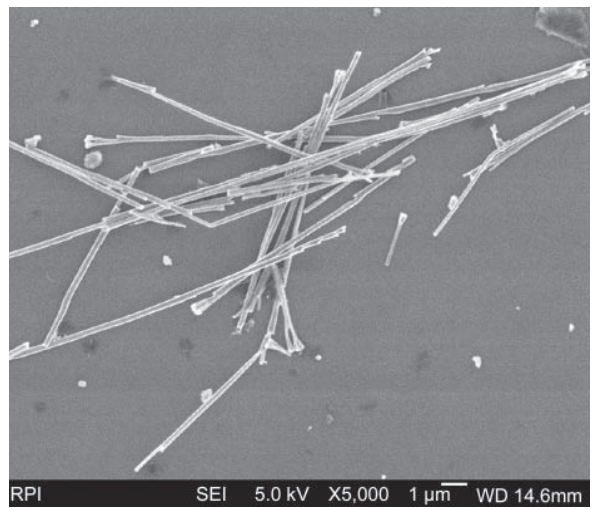

(b)

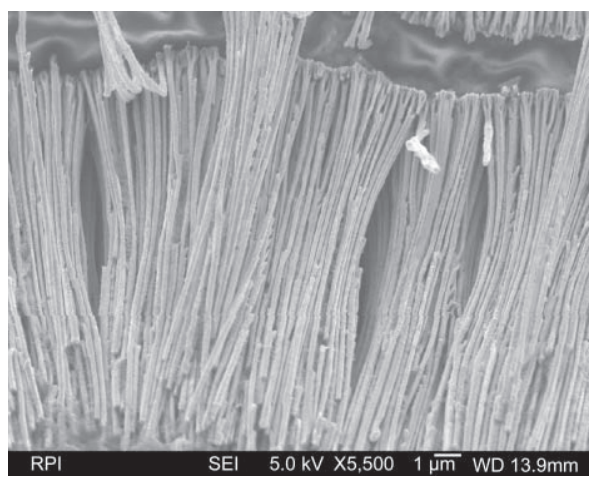

(c)

Figure 3 FESEM images of (a) Ni NW bundles after removal of alumina; (b) individual Ni NWs on a silicon substrate; (c) Co NW bundles after removal of alumina

are no other elemental impurities present in the nanowires. The peak corresponding to $\mathrm{Cu}(\sim 15 \%$ of $\mathrm{Ni}$ or $\mathrm{Co}$ ) arises from the $\mathrm{Cu}$ tape used for EDS measurements.

Transmission electron micrographs and selected area electron diffraction (SAED) of $\mathrm{Ni}$ NWs are shown in Fig. 5. It must be noted here that the samples were subjected to TEM studies after removing the template (alumina) using 3 


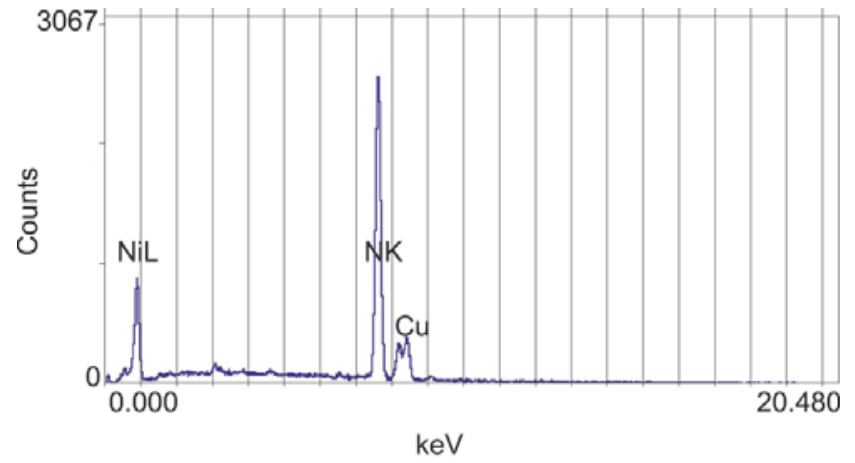

(a)

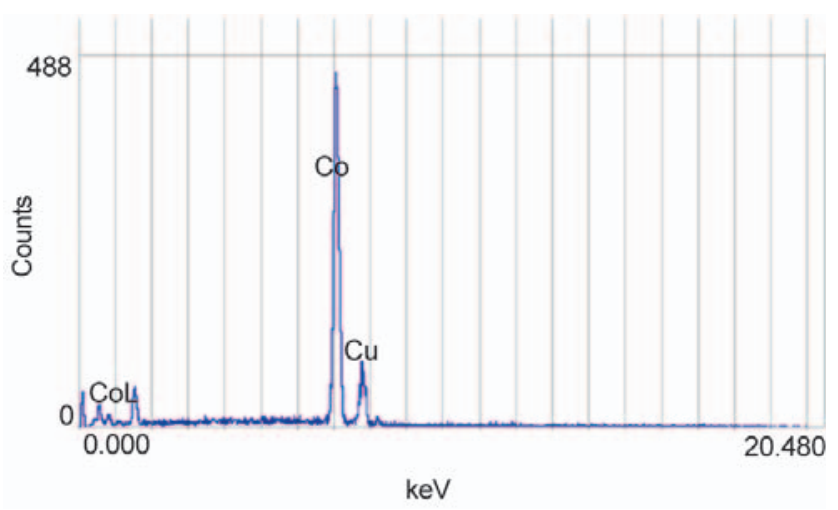

(b)

Figure 4 (a) EDS of Ni NWs; (b) EDS of Co NWs

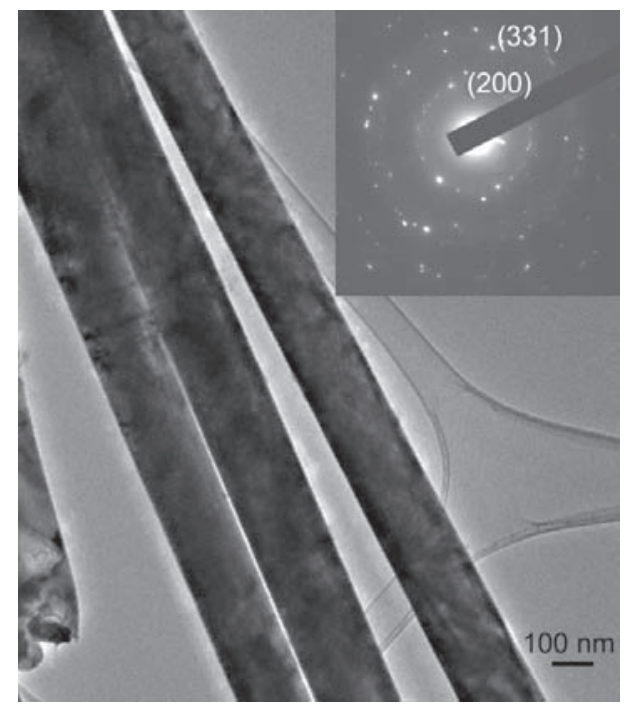

Figure 5 TEM image of Ni NWs, with (inset) SAED of Ni NWs

$\mathrm{mol} / \mathrm{L} \mathrm{NaOH}$ and the residue was magnetically separated. The residue was dissolved in ethanol and drop casted over a copper grid. The quality of the nanowires is evident from the micrograph. The SAED patterns (Fig. 5 inset) indicate that the nanowires are crystalline in nature.

\subsection{Magnetisation studies}

Magnetisation measurements ( $M-H$ measurements) were carried out using a SQUID magnetometer. Magnetisation studies of Ni NWs show features similar to those of nickel nanotubes [29], but the length of the nanowires in the present study is much larger and hence they exhibit a high shape anisotropy. The $M-H$ curves at $300 \mathrm{~K}$ and $6 \mathrm{~K}$ are shown in Figs. 6 and 7, respectively. Figure 6(b) represents a rescaled and expanded view of Fig. 6(a), highlighting the inand out- of plane coercivity differences. The loop parameters were evaluated and are shown in Table 1.

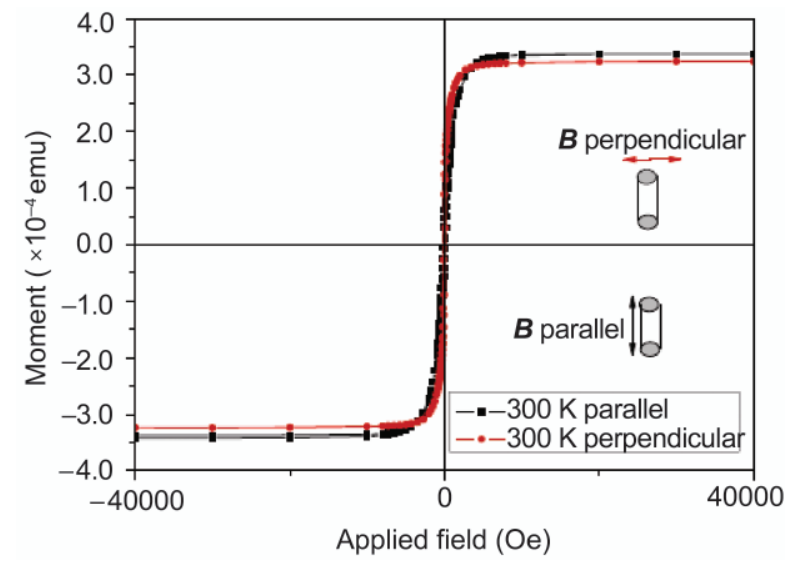

(a)

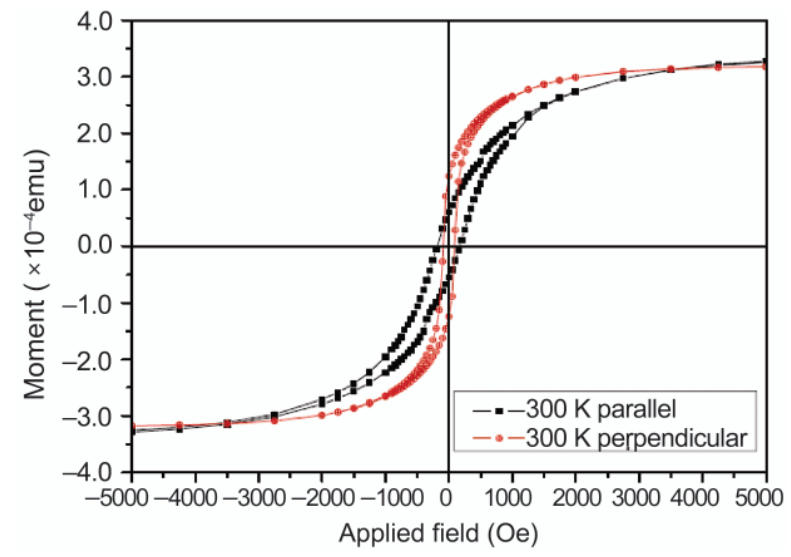

(b)

Figure 6 Magnetic hysteresis loops of Ni NWs at room temperature

Table 1 Hysteresis loop parameters for Ni NWs with two different field geometries at two different temperatures

\begin{tabular}{c|c|c|c|c}
\hline \multirow{2}{*}{$\begin{array}{c}\text { Temperature } \\
(\mathrm{K})\end{array}$} & \multicolumn{2}{|c|}{ Field parallel } & \multicolumn{2}{c}{ Field perpendicular } \\
\cline { 2 - 5 } & $H_{\mathrm{c}}(\mathrm{Oe})$ & $M_{\mathrm{r}} / M_{\mathrm{s}}$ & $H_{\mathrm{c}}(\mathrm{Oe})$ & $M_{\mathrm{r}} / M_{\mathrm{s}}$ \\
\hline 300 & 180 & 0.2 & 90 & 0.36 \\
\hline 6 & 260 & 0.1 & 220 & 0.2 \\
\hline
\end{tabular}




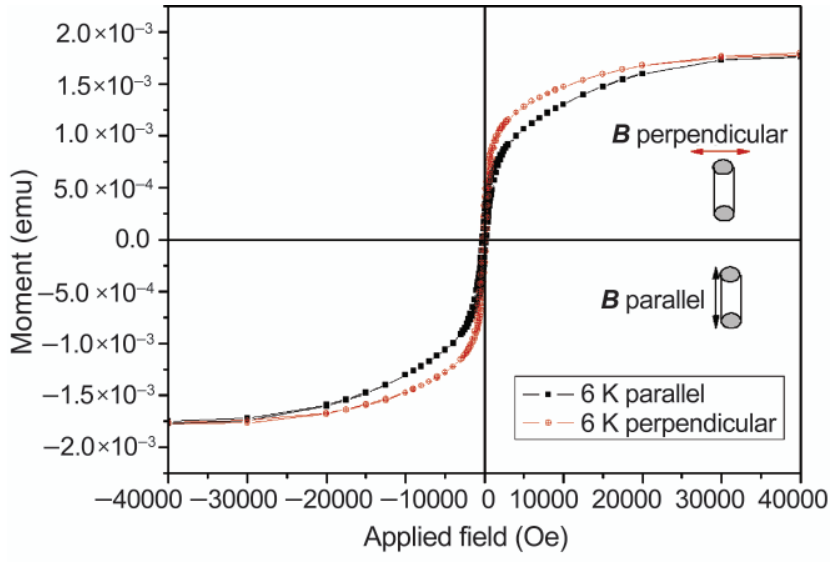

Figure 7 Hysteresis loop of Ni NWs at $6 \mathrm{~K}$

Coercivity of Ni nanowires $\left(H_{\mathrm{c}}\right.$ parallel and $H_{\mathrm{c}}$ perpendicular) exhibit enhanced values compared to that for the bulk Ni (around 0.7 Oe $[29,30]$ ). The coercivity values reported for nickel nanowires of similar diameter is $\sim 220$ Oe for longitudinal fields with a squareness value of 0.8 [31]. The squareness obtained for the parallel field is much less than the reported values for nickel nanowires. The crystal anisotropy $K_{1}$ of Ni NWs is $\sim 4 \times 10^{4} \mathrm{erg} / \mathrm{cm}^{3}$ and the shape anisotropy is $\pi M_{\mathrm{s}}^{2}=7 \times 10^{5} \mathrm{erg} / \mathrm{cm}^{3}$. Due to this large shape anisotropy and high aspect ratio ( 330), the easy magnetisation direction always lies along the wire axis. Moreover, for an fcc lattice the anisotropy orientation is pointing along the (110) direction. Therefore, from XRD and magnetisation measurements it is to be concluded that the anisotropy axis is aligned along the wire axis and it contributes to the shape anisotropy. Therefore, the easy axis is parallel to the wire axis. Previously reported [14] measurements on isoradial Ni NWs involved polycarbonate membranes or single crystal mica films where the typical pore density was $10^{-2}$ $\mu \mathrm{m}^{-2}$. In the case of the alumina template (used in the present study) the pore density lies in the range $10^{2}-$ $10^{3} \mu \mathrm{m}^{-2}$.

Figure 8 indicates that the inter-pore distance in the alumina template is $\sim 100 \mathrm{~nm}$. The high pore density in the case of alumina results in high interwire interactions which induce a dipolar field due to the adjacent wire interactions. This dipolar field will act as the demagnetization field, which is given by $\Delta H=-\Delta N \cdot M$, where $\Delta N$ is the demagnetization factor and $M$ is the magnetisation. The low $M_{\mathrm{r}} / M_{\mathrm{s}}$ ratio observed in the case of a parallel field, where it is expected to be $\sim 1$, is due to this high interwire interaction. Both the magnetisation curves are highly sheared due to this demagnetization field indicating strong interwire interactions, which is expected because the average separation between the nanowires is $\sim 100 \mathrm{~nm}$. The alumina template is not able to mediate exchange interactions over more than a few interatomic distances, so the interaction between the wires is realized only through magnetostatic dipolar interactions [32]. The effect of high dipolar interactions for perpendicular fields is to reduce the saturating field. The saturating field for a perpendicular field is $H_{\perp \mathrm{s}}=7 \mathrm{kOe}$ whereas that for a parallel field is $H_{\| \mathrm{s}}=10 \mathrm{kOe}$. The $M-H$ curve recorded at $6 \mathrm{~K}$ exhibits an increase in coercivity and a decrease in remanence parallel to the nanowire axis, consistent with an enhanced contribution of cubic magnetocrystalline anisotropy which is in competition with the uniaxial shape anisotropy [33]. Shape anisotropy is identical for low and high temperature measurements, because of the large length to diameter ratio $(\sim 330)$ [34].

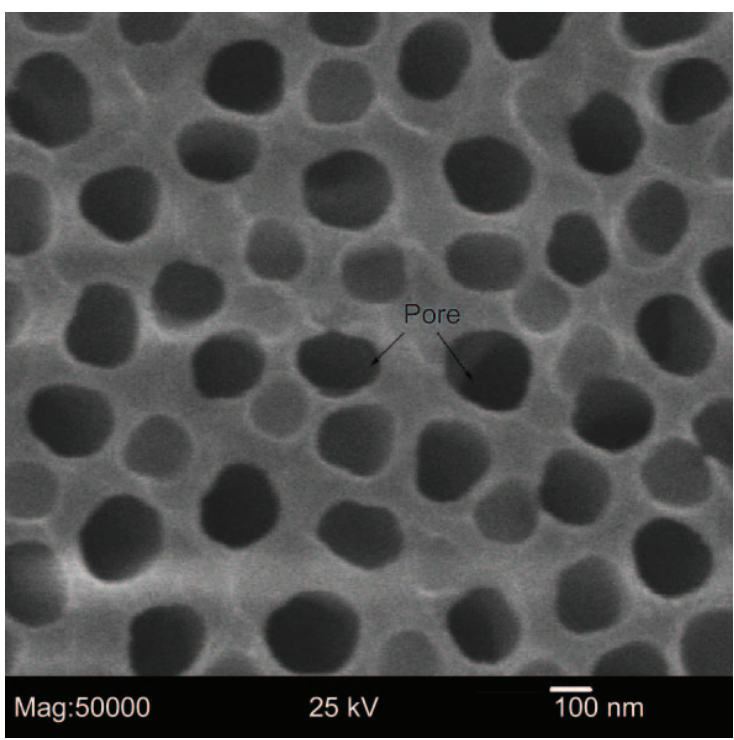

Figure 8 SEM image of the porous alumina template

Room temperature (300 K) and low temperature (6 K) magnetic properties of Co NWs are depicted in Figs. 9(a) and 9(b). The loops (parallel and perpendicular) are highly sheared and both exhibit a very low value of $M_{\mathrm{r}} / M_{\mathrm{s}}(\sim 0.1)$. This is perhaps due to high interwire 


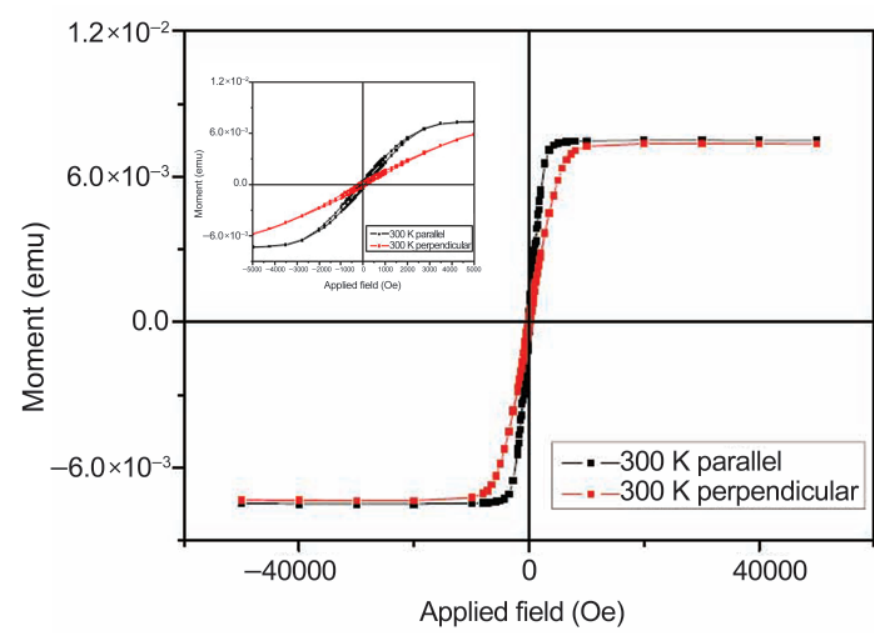

(a)

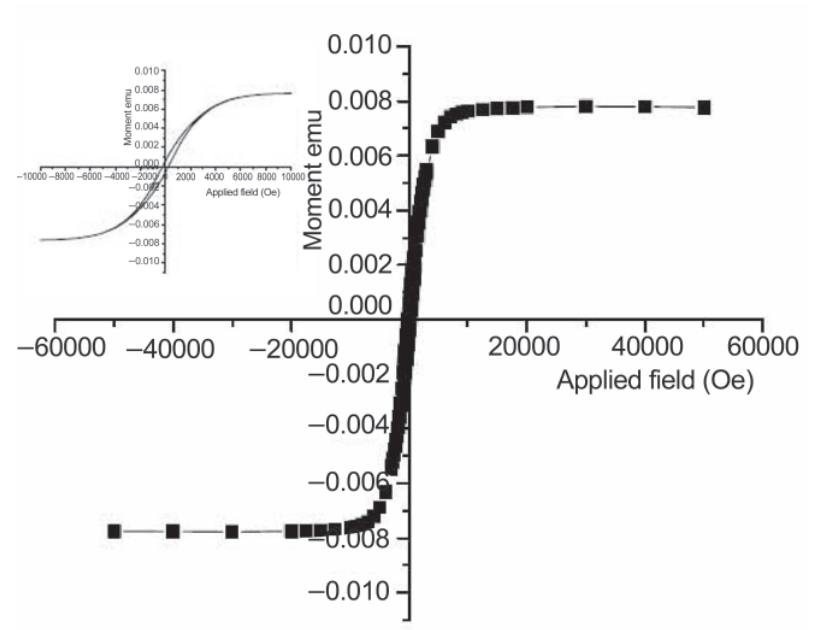

(b)

Figure 9 Hysteresis loops of Co NWs at (a) room temperature; (b) low temperature (field parallel to the wire)

interactions. Room temperature coercivity values of Ni NWs and Co NWs are by and large comparable. As in the case of Ni NWs, the low temperature coercivity of Co NWs exhibit an enhanced value $\left(H_{\mathrm{c} \mid} \sim 290\right.$ Oe) because of the higher magnetocrystalline anisotropy of these materials at low temperatures.

Figures 10(a) and 10(b) show the $M(T)$ measurement at $20 \mathrm{kOe}$ parallel to the nanowire. In this measurement the sample is cooled in zero field to low temperature $(5 \mathrm{~K})$. A field of $20 \mathrm{kOe}$ is then applied parallel to the wire and the $M(T)$ curve measured by warming up the sample in this field. Such a study enables one to compare the thermal demagnetization process with the field induced

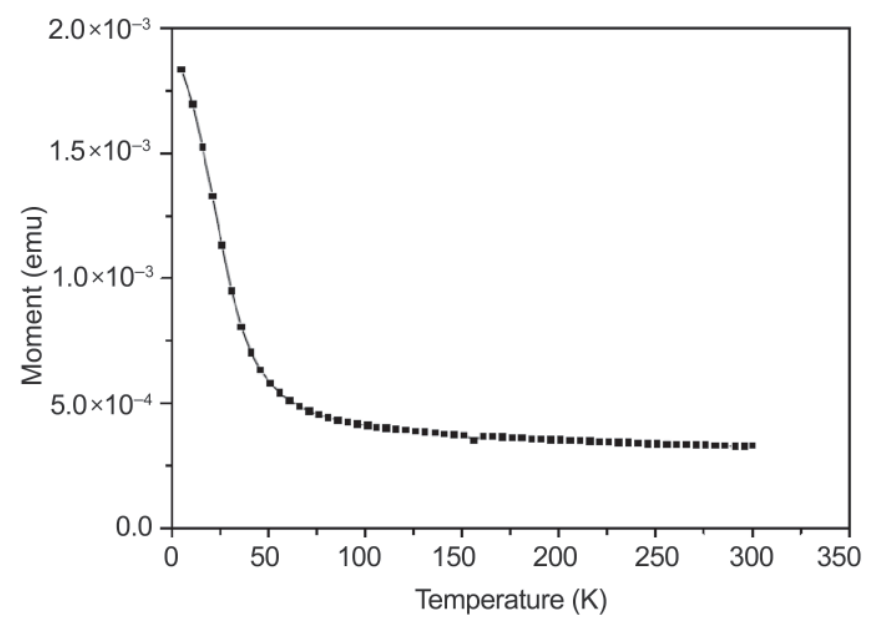

(a) reversal process at low temperatures. $M(T)$ curves show a switching of magnetisation from a high magnetisation value to a lower one ( one order change in magnetisation value) at a temperature $\sim 20 \mathrm{~K}$ during warming. Since the sample is ferromagnetic both at low and room temperatures as inferred from $M-H$ curves, this cooperative switching can only result from surface spin disorder. Since there are no indications of any oxide layer formation as evident from the XRD (Fig. 2), the chance of surface spin disorder resulting from antiferromagnetic interactions of nickel oxide is ruled out. The only possibility for such a switching of magnetisation in the parallel field measurements

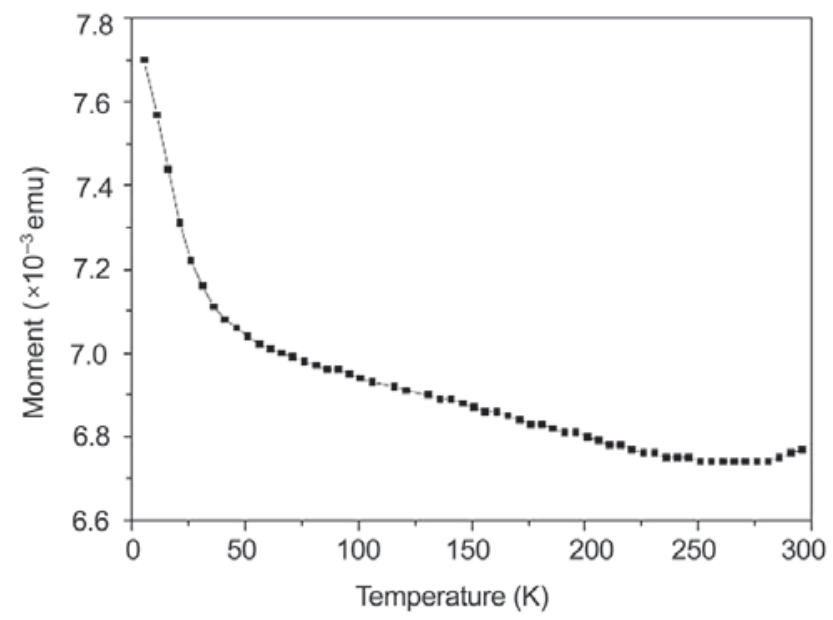

(b)

Figure $10 M(T)$ curves for (a) Ni NWs; (b) Co NWs, with a 20 kOe field applied parallel to the wire 
can be because of the dipolar interaction between adjacent nanowires. This helps to align the spin antiferromagnetically between adjacent wires. Magnetisation switching in terms of shape anisotropy is questionable and will not be complete in the case of wires where an interwire magnetostatic interaction exists. In the case of strong interwire interactions, the individual wires switch cooperatively and the behaviour is comparable to what happens in a thin film [35]. The M-T curves shown in Figs. 10(a) and 10(b) depict such a cooperative switching and this type of unusual $M-T$ curve also confirms the presence of strong interwire magnetostatic interactions in $\mathrm{Ni}$ and Co NWs.

\section{Conclusions}

High aspect ratio ( 330) nickel and cobalt nanowires have been prepared. The mobility assisted growth process responsible for nanowire formation is verified for both nickel and cobalt nanowires. This can be treated as a general growth mechanism in constant voltage deposition processes for all types of metal nanowires, and offers the possibility of controlling the formation of one-dimensional structures. Both $\mathrm{Ni}$ and Co NWs exhibit a preferential orientation along (220) and they are highly crystalline. A high interwire magnetostatic interaction was found to exist between wires and is manifested in low squareness values in the parallel field magnetisation measurements and low saturating field in the perpendicular field direction. The unusual high field $M(T)$ curves observed for both Ni and Co NWs are possibly due to the high dipolar interwire interaction. Thus, tuning of the magnetic properties of $\mathrm{Ni}$ and Co NWs is possible by engineering the interwire distances. This can be easily achieved by a template assisted synthesis. These highly crystalline textured $\mathrm{Ni}$ and Co NWs are possible candidates for perpendicular recording and various other multifunctional devices. Moreover, understanding the growth mechanism of onedimensional structures will facilitate the design of different coaxial multifunctional nanostructures which can find applications in various fields.

\section{Acknowledgements}

T. N. Narayanan acknowledges the financial support received from the Interconnect Focus Center at Rensselaer Polytechnic Institute, Troy, New York, USA. T. N. Narayanan thanks Kerala State Council for Science, Technology and Environment (D.O. No. 004/FSHIP/ 05/ KSCSTE), Kerala, India for financial support in the form of a fellowship. T. N. Narayanan and M. R. Anantharaman acknowledge Prof. Gunter Schatz, Prof. Manfred Albrecht, and Dr. Ildico Guhr, Department of Physics, University of Konstanz, Germany for SQUID measurements and fruitful discussions. M. R. Anantharaman thanks DST-DAAD PPP for awarding an exchange programme.

\section{References}

[1] Puntes, V. F.; Krishnan, K. M.; Alivisatos, A. P. Colloidal nanocrystal shape and size control: The case of cobalt. Science 2001, 291, 2115-2117.

[2] Jung, S. W.; Park, W. I.; Yi, G. C.; Kim, M. Y. Fabrication and controlled magnetic properties of $\mathrm{Ni} / \mathrm{ZnO}$ nanorod heterostructures. Adv. Mater. 2003, 15, 1358-1361.

[3] Bao, J.; Liang, Y.; Xu, Z.; Si, L. Facile synthesis of hollow nickel submicrometer spheres. Adv. Mater. 2003, 15, 1832-1835.

[4] Sahoo, S.; Petracic, O.; Kleemann, W.; Stappert, S.; Dumpich, G.; Nordblad, P.; Cardoso, S.; Freitas, P. P. Cooperative versus superparamagnetic behavior of dense magnetic nanoparticles in $\mathrm{CO}_{80} \mathrm{Fe}_{20} / \mathrm{Al}_{2} \mathrm{O}_{3}$ multilayers Appl. Phys. Lett. 2003, 82, 4116-4118.

[5] Garcia, J. M.; Asenjo, A.; Velazquez, J.; Garcia, D.; Vazquez, M.; Aranda, P.; Hitzky, E. R. Magnetic behavior of an array of cobalt nanowires. J. Appl. Phys. 1999, 85, 5480-5482.

[6] Martin, J. I.; Nogues, J.; Liu, K.; Vicent, J. L.; Schuller, I. K. Ordered magnetic nanostructures: Fabrication and properties. J. Magn. Magn. Mater. 2003, 256, 449-451.

[7] Yu, C. Y.; Yu, Y. L.; Sun, H. Y.; Xu, T.; Li, X. H.; Li, W.; Gao, Z. S.; Zhang, X. Y. Enhancement of the coercivity of electrodeposited nickel nanowire arrays. Mater. Lett. 2007, 61, 1859-1862.

[8] Whitney, T. M.; Searson, P. C.; Jiang, J. S.; Chien, C. L. Fabrication and magnetic properties of arrays of metallic nanowires. Science 1993, 261, 1316-1319.

[9] Basu, S.; Chatterjee, S.; Saha, M.; Bandyopadhyay, M.; 
Mistry, K.; Sengupta, K. Study of electrical characteristics of porous alumina sensors for detection of low moisture in gases. Sens. Actuat. B-Chem. 2001, 79, 182-185.

[10] Meng, G.; Cao, A.; Cheng, J. Y.; Vijayaraghavan, A.; Jung, Y. J.; Shima, M.; Ajayan, P. M. Ordered Ni nanowire tip arrays sticking out of the anodic aluminum oxide template. J. Appl. Phys. 2005, 97, 064303.

[11] Lew, K. K.; Redwing, J. Growth characteristics of silicon nanowires synthesized by vapor-liquid-solid growth in nanoporous alumina templates. J. Cryst. Growth 2003, 254, 14-17.

[12] Wang, Y. C.; Leu, I. C.; Hon, M. H. Preparation and characterization of nanosized $\mathrm{ZnO}$ arrays by electrophoretic deposition. J. Cryst. Growth 2002, 237, 564-567.

[13] Athanssiou, E. K.; Grossmann, P.; Grass, R. N.; Stark, W. J. Template free, large scale synthesis of cobalt nanowires using magnetic fields for alignment. Nanotechnology 2007, 18, 165606.

[14] Fert, A.; Piraux, L. Magnetic nanowires. J. Magn. Magn. Mater. 1999, 200, 338-358.

[15] Fasol, G. Nanowires: Small is beautiful. Science 1998, 280, 545-546.

[16] Martin, C. R. Nanomaterials: A membrane-based synthetic approach. Science 1994, 266, 1961-1966.

[17] Kodama, R. H.; Berkwitz, A. E.; Meniff, E. J.; Foner, S. Surface spin disorder in $\mathrm{NiFe}_{2} \mathrm{O}_{4}$ nanoparticles. Phys. Rev. Lett. 1996, 77, 394-397.

[18] Goya, G. F.; Forseca, F. C.; Jardin, R. F.; Muceillo, R.; Carreno, N. L. V.; Longo, E.; Leite, E. R. Magnetic dynamics of single-domain Ni nanoparticles. J. Appl. Phys. 2003, 93, 6531-6533.

[19] Kafil, M. R.; Saibal, R. Thermal diffusivity of nonfractal and fractal nickel nanowires. J. Appl. Phys. 2008, 103, 084302.

[20] Escrig, J.; Lavin, R.; Palma, J. L.; Denardin, J. C.; Altbir, D.; Cortes, A.; Gomez, H. Geometry dependence of coercivity in Ni nanowire arrays. Nanotechnology 2008, 19, 075713.

[21] Rivas, J.; Kazadi Mukenga Bantu, A.; Zaragoza, G.; Blanco, M. C.; Lopez-Quintela, M. A. Preparation and magnetic behavior of arrays of electrodeposited Co nanowires. J. Magn. Magn. Mater. 2002, 249, 220-225.

[22] Gubbiotti, G.; Tacchi, S.; Carlotti, G.; Vavassori, P.; Singh, N.; Goolaup, S.; Adeyeye, A. O.; Stashkevich, A.; Kostylev, M. Magnetostatic interaction in arrays of nanometric permalloy wires: A magneto-optic Kerr effect and a Brillouin light scattering study. Phys. Rev. B 2005,
$72,224413$.

[23] Goolaup, S.; Adeyeye, A. O.; Singh, N. Dipolar coupling in closely packed pseudo-spin-valve nanowire arrays. J. Appl. Phys. 2006, 100, 114301.

[24] Ou, F. S.; Shaijumon, M. M.; Ajayan, P. M. Controlled manipulation of giant hybrid in-organic assemblies. Nano Lett. 2008, 8, 1853-1857.

[25] Narayanan, T. N.; Shaijumon, M. M.; Ajayan, P. M.; Anantharaman, M. R. Synthesis of high coercivity cobalt nanotubes with acetate precursors and elucidation of the mechanism of growth. J. Phys. Chem. C 2008, 112, 14281-14285.

[26] Bantu, A. K. M.; Rivas, J.; Zaragoza, G.; Quintela, M. A. L.; Blanco, M. C. Structure and magnetic properties of electrodeposited cobalt nanowires. J. Appl. Phys. 2001, 89, 3393-3397.

[27] Cao, H.; Wang, L; Qiu, Y.; Wu, Q.; Wang, G.; Zhang, L.; Liu, X. Generation and growth mechanism of metal (Fe, Co, Ni) nanotube arrays. ChemPhysChem 2006, 7, 1500 -1504 .

[28] Cao, H.; Tie, C.; Xu, Z.; Hong, J.; Sang, H. Array of nickel nanowires enveloped in polyaniline nanotubules and its magnetic behavior. Appl. Phys. Lett. 2001, 78, 1592-1594.

[29] Bao, J.; Tie, C.; Xu, Z.; Zhou, Q.; Shen, D.; Ma, Q. Template synthesis of an array of nickel nanotubules and its magnetic behavior. Adv. Mater. 2001, 13, 1631-1633.

[30] Chikazumi, S. Physics of Magnetism; John Wiley \& Sons: New York, 1964.

[31] Sun, L.; Hao, Y.; Chein, C. L.; Searson, P. C. Tuning the properties of magnetic nanowires. IBM J. Res. Dev. 2005, 49, 79-102.

[32] Sellmyer, D. J.; Zheng, M.; Skomski, R. Magnetism of Fe, $\mathrm{Co}$ and $\mathrm{Ni}$ nanowires in self-assembled arrays. J. Phys.: Condens. Mat. 2001, 13, R433-R460.

[33] Heydon, G. P.; Hoon, S. R.; Farley, A. N.; Tomlinson, S. L.; Valera, M. S.; Attenborough, K.; Schwarzacher, W. Magnetic properties of electrodeposited nanowires. J. Phys. D: Appl. Phys. 1997, 30, 1083-1093.

[34] Henry, Y.; Ounadjela, K.; Piraux, L.; Dubois, S.; George, J. M. ; Duvail, J. L. Magnetic anisotropy and domain patterns in electrodeposited cobalt nanowires. Eur. Phys. J. B 2001, 20, 35-54.

[35] Ounadjela, K.; Ferre, R.; Louail, L.; George, J. M.; Maurice, J. L.; Pirax, L.; Dubuis, S. Magnetization reversal in cobalt and nickel electrodeposited nanowires. J. Appl. Phys. 1997, 81, 5455-5457. 